\title{
Scaling collapse at the jamming transition
}

\author{
Yoav Kallus \\ Santa Fe Institute, 1399 Hyde Park Road, Santa Fe, New Mexico 87501
}

(Dated: June 25, 2021)

\begin{abstract}
The jamming transition of particles with finite-range interactions is characterized by a variety of critical phenomena, including power law distributions of marginal contacts. We numerically study a recently proposed simple model of jamming, which is conjectured to lie in the same universality class as the jamming of spheres in all dimensions. We extract numerical estimates of the critical exponents, $\theta=0.451 \pm 0.006$ and $\gamma=0.404 \pm 0.004$, that match the exponents observed in sphere packing systems. We analyze finite-size scaling effects that manifest in a subcritical cutoff regime and size-independent, but protocol-dependent scaling curves. Our results supports the conjectured link with sphere jamming, provide more precise measurements of the critical exponents than previously reported, and shed light on the finite-size scaling behavior of continuous constraint satisfiability transitions.
\end{abstract}

Jamming is the continuous emergence of nonzero mechanical moduli in a system of particles with finiterange interactions $\mathrm{BBB}^{+} 11$, TS10, OLLN02. The ensemble of configurations at the transition boundary exhibits many dramatic phenomena, including anomalous vibration spectra WWW05, Par14, FPUZ15, isostaticity and hyperuniformity ZJT11, critical scaling of mechanical moduli vH09, and power-law distributions of the weakest forces and the smallest interparticle gaps CCPZ12, FP15, KMT13. The jamming transition can be understood as a continuous analog of the satisfiability transition in random constraint satisfaction problems [FP15, usually studied in the discrete setting [PIM06]. Recently the transition in a simple constraint problem, the perceptron, was conjectured to lie in the same universality class as the sphere jamming transition in all spatial dimensions [FP15]. Here we numerically study the perceptron and show that the power law exponents match those measured numerically for sphere jamming, but may not match the theoretically predicted exponents. We employ finite-size scaling to extract precise measurements of the exponents and demonstrate the universal critical behavior of the system. Our results support the conjecture linking the perceptron to sphere jamming, and provide more precise numerical estimates of the jamming critical exponents than previously reported.

The jamming ensemble corresponds to the random packing configurations of nonoverlapping hard spheres such that no improvement in the packing density is possible by local movement of the spheres. As such, it is a local transition between the satisfiability of the nonoverlap constraints at lower density, and unsatisfiabily of all constraints simultaneously at higher density. This transition for equal-sized three-dimensional spheres occurs typically at a range of densities around $64 \%$ depending on preparation protocol CBS10. The restriction to random packing is crucial, since the constraints are simultaneously satisfiable all the way to the close packing density of spheres, $74 \%$. As a result, any theoretical treatment of the jamming transition must overcome the problem of excluding configurations with crystalline order from the phase space. One approach that has been successful in reproducing the metastable disordered phase behavior of systems with a thermodynamically stable ordered phase has been to construct systems with quenched disordered that exhibit the same correlation structure in the disordered phase [MPR94].

The perceptron model, well known in machine learning Ros58, when extended to its nonconvex regime, has been shown by Franz and Parisi to be solved by the same replica symmetry breaking (RSB) ansatz as that occurring as a solution for the jamming point in a model of sphere packing in the limit of an infinite number of dimensions [FP15, $\left.\mathrm{CKP}^{+} 14\right]$. It is conjectured that the jamming transitions of spheres in all dimensions lie in the same universality class as these two models CCPZ12, $\mathrm{CKP}^{+} 14$. Here, we numerically simulate the perceptron model and compare our numerical results to numerical simulations of sphere jamming and the theoretical predictions of the RSB solution.

For a given size $n$ and number of constraints $m$, an instance of the perceptron is given by the following constrained optimization problem:

$$
\begin{aligned}
& \operatorname{maximize} F(\mathbf{x})=\|\mathbf{x}\|^{2}=\langle\mathbf{x}, \mathbf{x}\rangle \\
& \text { subject to } \mathbf{x} \in \mathbb{R}^{n} \\
& \qquad\left\langle\xi_{\mu}, \mathbf{x}\right\rangle \leq 1 \text { for all } \mu=1, \ldots, m,
\end{aligned}
$$

where $\xi_{\mu}, \mu=1, \ldots, m$, are independently drawn from a uniform distribution over the unit sphere $S^{n-1}$. To approach the thermodynamic limit, the system size $n$ is increased toward infinity while the ratio $\alpha=m / n$ is held constant FP15.

An equivalent formulation, closer to the traditional setting of the perceptron model is obtained by restricting $\mathbf{x}$ to $S^{n-1}$ and maximizing the distance from the nearest point $\xi_{\mu}$. However, the formulation (1) allows all the constraints to be linear inequality constraints. Thus, the only nonlinearity, distinguishing (1) from a linear program (LP), is in the objective function. Moreover, since the objective is convex, the local maxima occur at vertices of the feasible polytope, so the number of active constraints (constraints satisfied with equality) must be at least $n$. (It can also be no greater than $n$ due to 


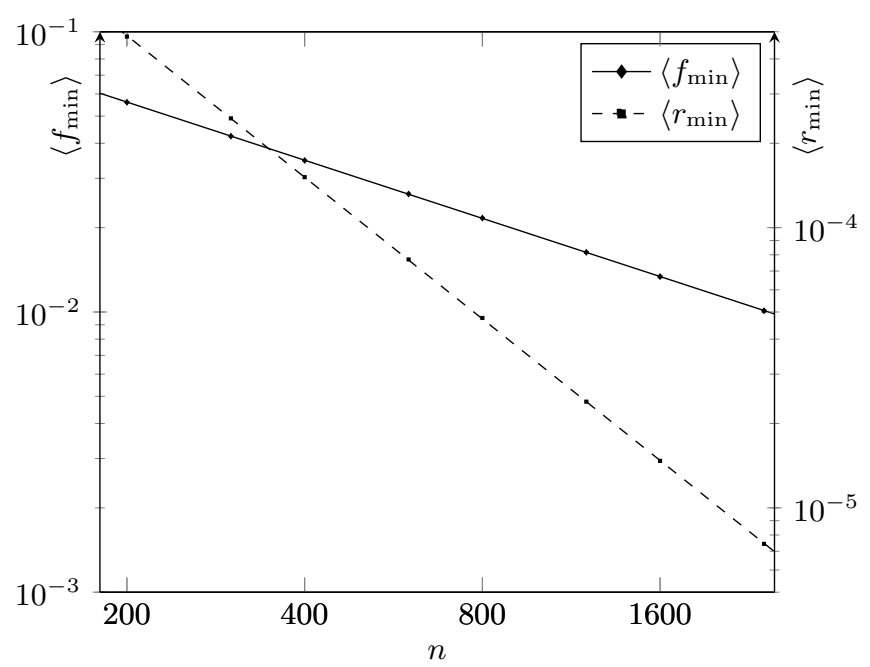

FIG. 1. Mean values of the smallest force (solid line) and of the smallest slack (dashed line) in the locally optimal solutions reached by the sequential LP protocol in random instances of the perceptron as a function of system size. The lines show the power-law fit to the data, from which we extract the numerical exponent estimates: $\theta=0.451 \pm 0.006$ and $\gamma=0.404 \pm 0.004$.

the genericity of the constraints). In these properties, the model is reminiscent of an earlier jamming model, namely the jamming transition of lattice sphere packing KMT13, KT14. In this model, the determinant of a symmetric $d \times d$ matrix $Q$ is minimized, subject to the linear constraints $\langle\xi, Q \xi\rangle \geq 1$ for all $\xi \in \mathbb{Z}^{d}$. Whereas the perceptron model has quenched disorder, the sphere packing and lattice packing models do not and have an ordered phase in addition to the disordered phase.

One of the remarkable properties of the jamming ensemble observed in sphere jamming is the power law distribution of very weak contact forces and of very small interparticle gaps. In the context of general constrained optimization problems, these correspond to the distribution of Lagrange (or Karush-Kuhn-Tucker) multipliers associated with active inequality constraints and the distribution of slacks of inactive constraints (the difference between the two sides of an inequality constraint). The constraints at the low end of each of these distributions can be thought of as the marginal constraints: the active constraints closest to becoming inactive and vice versa. The observation of power law distributions of marginal constraints has been explained heuristically as resulting from the marginal stability of typical jammed configuration Wya12]: in order for the configuration to be stable, it must either exhibit a suppression of weak active constraints, which contribute to instability, or an enhancement of small-slack inactive constraints, which contribute to stability, or both, as is observed. A quantitative version of this argument gives a predicted relation between the exponents of the two power laws.

At a locally optimal feasible point of (1) standard results in nonlinear optimization imply the existence of La-

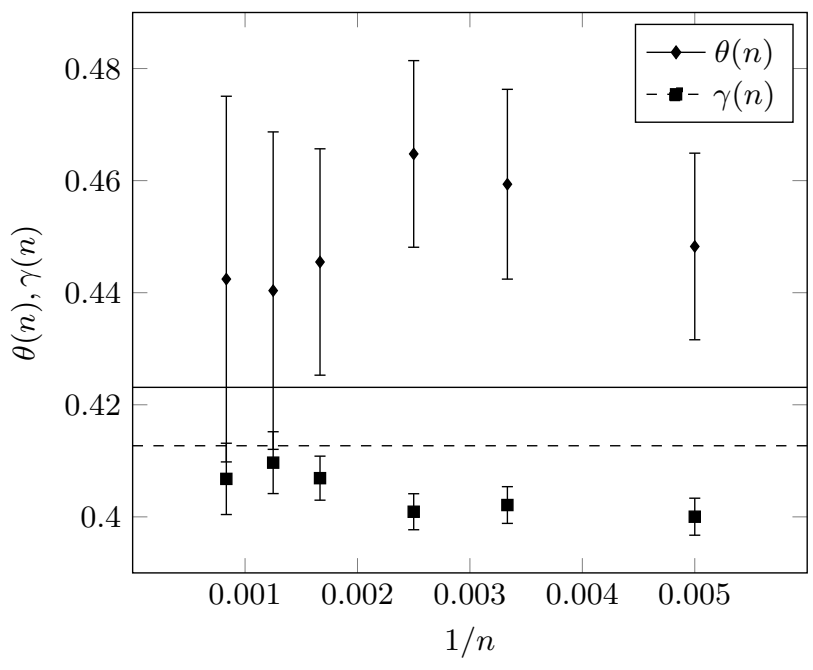

FIG. 2. Critical exponent values inferred from the average slopes of the curves of Figure 1 measured between between $n$ and $2 n$. The horizontal lines are the theoretically predicted values. No clear trend signaling corrections to finite-size scaling is apparent.

grange multipliers (forces) $f_{\mu}$, such that $f_{\mu}>0$ if and only if $\left\langle\xi_{\mu}, \mathbf{x}\right\rangle=1$, and $\mathbf{x}=\|\mathbf{x}\| n^{-1 / 2} \sum_{\mu} f_{\mu} \xi_{\mu}$. It is predicted that in the thermodynamic limit, the distribution of forces satisfies a power law $p(f) \sim f^{\theta}$ with a universal exponent $\theta=0.42311$ for small $f$ FP15]. In particular, the smallest force is of the order of the value $f_{\min }$ such that $\int_{0}^{f_{\min }} p(f) d f \sim \frac{1}{n}$. That is, $f_{\min } \sim n^{-1 /(1+\theta)}$. For finite systems, we expect the distribution to follow the power law only in the range $n^{-1 /(1+\theta)} \ll f \ll 1$. However, as in other universal critical points, we expect a finite-size scaling behavior even for moderately sized finite systems. In particular, we expect, when $f \ll 1$,

$$
p(f)=n^{-\theta /(1+\theta)} \tilde{p}\left(f n^{1 /(1+\theta)}\right) .
$$

We are also interested in the distribution of slacks of inactive constraints. Let $r_{\mu}=n^{1 / 2}\left(1-\left\langle\xi_{\mu}, \mathbf{x}\right\rangle\right) /\|\mathbf{x}\|$ and let $n g(r) d r$ be the expected number of constraints $\mu$ such that $0<r \leq r_{\mu} \leq r+d r$. The theoretically predicted distribution for small $r$ in the thermodynamic limit is $g(r) \sim r^{-\gamma}$, and consequently the smallest nonzero slack should scale with system size as $r_{\min } \sim n^{-1 /(1-\gamma)}$ [FP15]. The theoretically predicted universal value for the exponent is $\gamma=0.41269$ [FP15]. For finite systems, the power law should be cut off around $r_{\text {min }}$ consistent with a finitesize scaling function

$$
g(r)=n^{\gamma /(1-\gamma)} \tilde{g}\left(r n^{1 /(1-\gamma)}\right) .
$$

The marginal stability heuristic predicts that $\gamma=1 /(2+$ $\theta)$, a relationship that is satisfied by the theoretically predicted values Wya12, FP15.

We perform numerical optimization of the perceptron at a constraint ratio $\alpha=4$ and sizes $n=200,300,400$, 

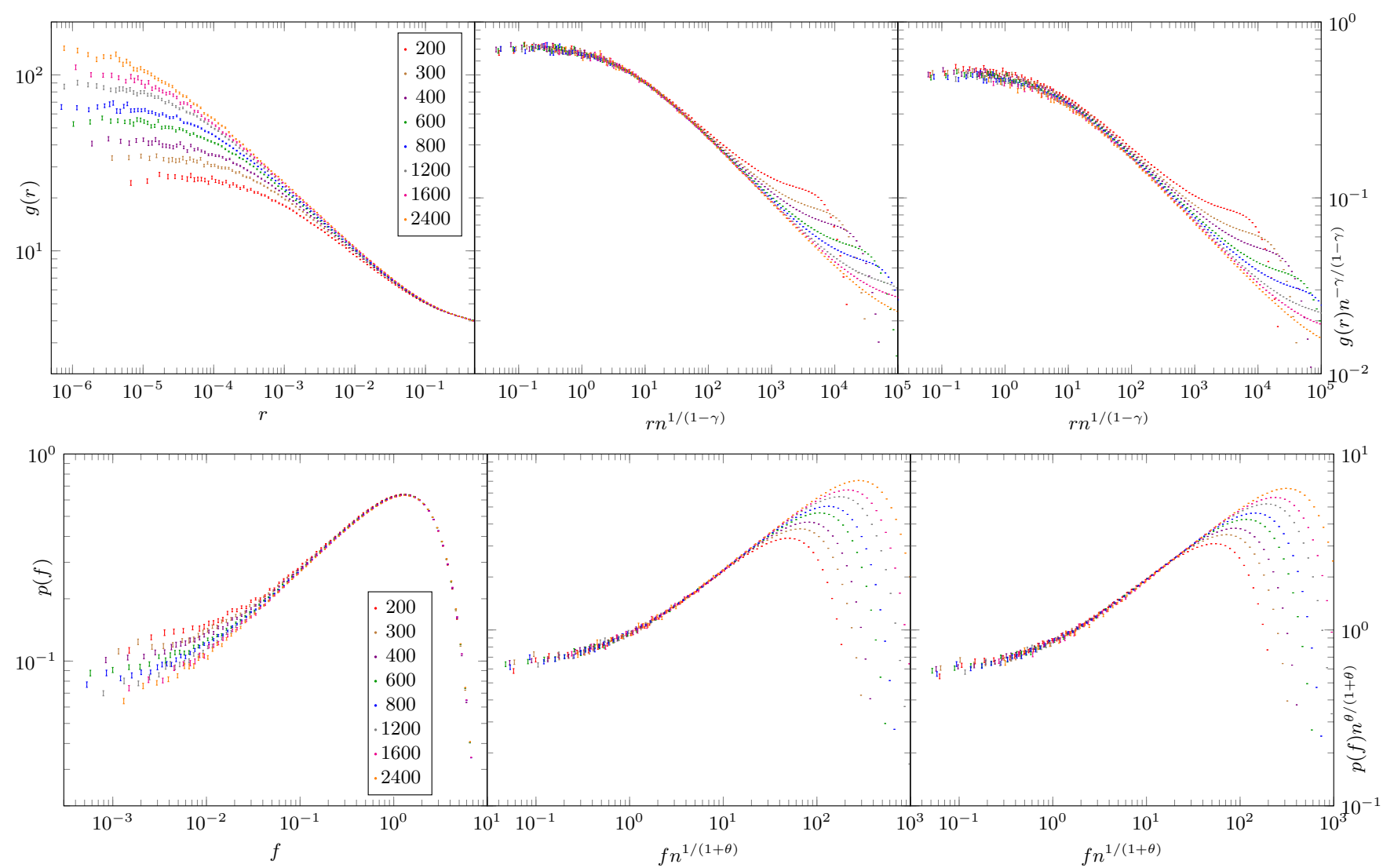

FIG. 3. Empirical distributions (left) of slacks of inactive constraints (top) and Lagrange multipliers of active constraints (bottom) at the jamming point. The middle and right panels show the finite-size scaling collapse of the distributions using the numerically extracted exponents and the theoretically predicted exponents respectively.

$600,800,1200,1600$, and 2400 . We generate $10^{5}, 10^{5}$, $10^{5}, 10^{5}, 10^{5}, 5 \cdot 10^{4}, 2 \cdot 10^{4}$, and $2 \cdot 10^{4}$ independent realizations, respectively, of each size. The optimization protocol solves a sequence of LPs that are identical to (1). except that the objective is replaced by a linear objective $F_{t}(\mathbf{x})=\left\langle\mathbf{y}_{t}, \mathbf{x}\right\rangle$. The objective vector for each step is given by the solution of the previous LP: $\mathbf{y}_{t+1}=\mathbf{x}_{t}^{\text {opt }}$. The real objective $\|\mathbf{x}\|^{2}$ necessarily increases at each step and the process converges to a local optimum of (1) in finitely many steps.

In Figure 1, we plot the mean value of the smallest force $\left\langle f_{\min }\right\rangle$ and the mean value of the smallest slack $\left\langle r_{\min }\right\rangle$ as functions of the system size. In both cases, we obtain a power-law fit as predicted. The inferred values of the critical exponents are $\gamma=0.404 \pm 0.004$ and $\theta=0.451 \pm 0.006$. The inferred values are close to the theoretically predicted values, but do not seem to include the latter in their confidence regions. Still, the inferred values satisfy the marginality relation $\gamma=1 /(2+\theta)$.

It is possible that corrections to finite-size scaling are responsible for the discrepancy between our numericallyinferred exponents and the theoretical values and that the apparent exponents at finite $n$ are different from their limit as $n \rightarrow \infty$. We measure the local slopes of the curves of Figure 1, and we do not observe a clear trend in $\gamma(n)$ or $\theta(n)$, which would be indicative of corrections to finite-size scaling (see Figure 2 . Still, partly due to the increase in the error estimate, the slope values at larger sizes seem to be more consistent with the theoretically predicted exponents. Another explanation could be that the thermodynamic calculation predicting the exponent values only approximately applies to the setting at hand, which employs a highly off-equilibrium hill-climbing dynamic.

Previous numerical studies of systems conjectured to be in the same universality class provide similar estimates of the critical exponents. Studying hard and soft spheres in up to 10 dimensions, the authors of Ref. CCPZ12 find $\gamma \approx 0.39$ for soft spheres, and $\gamma \approx 0.42$ for hard spheres. In finite-dimensional sphere packings, the distribution of contact forces has additional contributions due to localized modes that dominates at low forces, but the relevant exponent $\theta_{e}$ can be extracted by looking only at contacts coupled to extended modes LDW13, CCPZ15. In Ref. LDW13, the authors measure $\gamma \approx 0.38$ and $\theta_{e} \approx 0.44$ for hard spheres in 3 dimensions. In Ref. [DLBW14, the authors measure $\gamma \approx 0.4$ and $\theta_{e} \approx 0.44$ for hard spheres in 2 and 3 dimensions. These values closely agree with our estimates of the critical exponents in the perceptron. Since we approach the transition from the satisfiable side, 


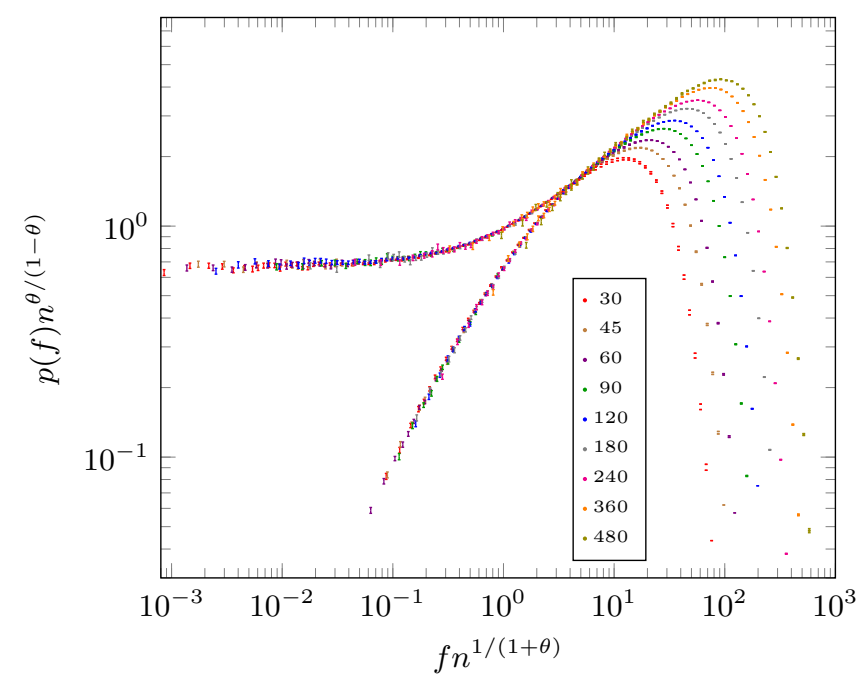

FIG. 4. Finite-size scaling collapse for jamming points of the edge-climbing and edge-jumping protocols. The two sets of data collapse to a common curve in the critical regime, but to two separate scaling curves in the subcritical regime.

our results should be considered analogous to the hard sphere results.

We now turn to inspecting the distributions $g(r)$ and $p(f)$ presented in Figure 3 . As the system size increases, a clear power law develops extending to lower and lower values of $r$ and $f$. However, at each finite size, the power law is cut off and approaches a constant as $r, f \rightarrow 0$. The finite nonzero values of $g(0)$ and $p(0)$, are of particular interest. Observation of such cutoffs have previously been cited as evidence against the existence of a power law (or for trivial exponents $\gamma=\theta=0$ ) DTS05, TSS $^{+} 05$, TSVvH10, KT14, BMSM14, but in the current context can be understood as finite-size effects.

The region controlled entirely by the power law appears to be no more than two decades of magnitude at the largest system size for each distribution. Therefore, even when we use the steepest decade of data at the largest system size to fit a power law, we obtain exponent estimates significantly lower than the ones inferred by comparing different system sizes: $\gamma \approx 0.37$ and $\theta \approx 0.42$. The shallower behavior in the cutoff regimes affects the curve significantly even dacades away from the overt crossover. This appears to not be the case for sphere jamming, where the sparsity of the constraints in comparison with the number of degrees of freedom seems to allow the efficient simulation of much larger systems, where the power-law regime spans 3-4 decades. Even in that case, the subcritical and supercritical regimes could still have an effect on the apparent power-law exponents.
Studying the finite-size scaling of the distribution could provide a useful comparison.

We can test the finite-size scaling hypotheses, (2) and (3), by appropriately rescaling the axes by the appropriate powers of $n$. When we use our inferred exponent values to rescale the data (see Figure 3), they collapse to a size-independent curve, with a common behavior in the critical and subcritical regimes. The theoretically predicted exponent values give a slightly, but visibly, poorer collapse, especially for the distribution of slacks.

While the scaling curve appears to be size independent, we do observe striking protocol dependence: in addition to the sequential LP protocol described above, we implemented two additional protocols at smaller sizes. The edge-climbing (EC) protocol mimics the simplex algorithm of linear programming, starting at a vertex of the feasible region and traveling at each step to an adjacent vertex along the edge offering the largest directional derivative of the objective. When all such derivatives are negative, a local optimum is achieved and the protocol terminates. The edge-jumping (EJ) protocol makes EC moves when they are available, but otherwise tries each edge and follows it if the objective value at the other endpoint is larger. If neither type of move is available, the protocol terminates.

We see an overt difference in the distribution of forces at the jamming point achieved by the two protocols Figure 4). While $p(f)$ shows similar behaviors in the power law regime, below the cutoff we observe $p(f) \sim$ constant in the EC protocol, and $p(f) \sim f$ in the EJ protocol. The edge-jumping moves destabilize weak constraints and suppress the subcritical regime of the force distribution in a nonuniversal way, clearly distinguished from the universal critical suppression.

The perceptron model provides a numerically tractable setting in which to test many of the theoretical ideas and methods about the jamming transition. Finite-size effects studied here could be crucial to applying these ideas to real-world problems away form the thermodynamic limit. In the present case at least, comparison of systems of different sizes provides a more sensitive measure of the critical exponents, which are distorted by finite-size effects in any single size studied here. Our estimates of the critical exponents match those measured numerically in sphere jamming systems, supporting the conjecture that they are in the same universality class. However, we observe some variance from the theoretically predicted exponent values, both in the scaling of the minimal force and minimal slack with system size, and in the finite-size scaling collapse. Finally, we observe size-independent but protocol-dependent finite-size scaling curves that demonstrate distinct critical and subcritical behavior.

Acknowledgment. This work was supported by an Omidyar Fellowship at the Santa Fe Institute. 
ford Univ Press, Oxford, 2011.

[BMSM14] L. Bo, R. Mari, C. Song, and H. A. Makse. Cavity method for force transmission in jammed disordered packings of hard particles. Soft Matter, 10:7379-7392, 2014.

[CBS10] P. Chaudhuri, L. Berthier, and S. Sastry. Jamming transitions in amorphous packings of frictionless spheres occur over a continuous range of volume fractions. Phys. Rev. Lett., 104:165701, 2010.

[CCPZ12] P. Charbonneau, E. I. Corwin, G. Parisi, and F. Zamponi. Universal microstructure and mechanical stability of jammed packings. Phys. Rev. Lett., 109(20), 2012.

[CCPZ15] P Charbonneau, E. I. Corwin, G. Parisi, and F. Zamponi. Jamming criticality revealed by removing localized buckling excitations. Phys. Rev. Lett., 114:125504, 2015.

$\left[\mathrm{CKP}^{+}{ }^{14}\right]$ P. Charbonneau, J. Kurchan, G. Parisi, P. Urbani, and F. Zamponi. Exact theory of dense amorphous hard spheres in high dimension III: The full replica symmetry breaking solution. J. Stat. Mech., 2014(10):P10009, 2014.

[DLBW14] E. DeGiuli, E. Lerner, C. Brito, and M. Wyart. Force distribution affects vibrational properties in hard-sphere glasses. Proc. Natl. Acad. Sci. USA, 111(48):17054-17059, 2014.

[DTS05] A. Donev, S. Torquato, and F. H. Stillinger. Pair correlation function characteristics of nearly jammed disordered and ordered hard-sphere packings. Phys. Rev. E, 71:011105, 2005.

[FP15] S. Franz and G. Parisi. The simplest model of jamming. arXiv:1501.03397, 2015.

[FPUZ15] F. Franz, G. Parisi, P. Urbani, and F. Zamponi. Universal spectrum of normal modes in low-temperature glasses: an exact solution. Proc. Nat. Acad. Sci. USA, 112(47):1453914544, 2015.

[KMT13] Y. Kallus, É. Marcotte, and S. Torquato. Jammed lattice sphere packings. Phys. Rev. E, 88(6):062151, 2013.

[KT14] Y. Kallus and S. Torquato. Marginal stability in jammed packings: Quasicontacts and weak contacts. Phys. Rev. E, 90(2):022114, 2014.

[LDW13] E. Lerner, G. Düring, and M. Wyart. Low-energy non-linear excitations in sphere packings. Soft Matter, 9:8252-8263, 2013.

[MPR94] E. Marinari, G. Parisi, and F. Ritort. Replica field theory for deterministic models: II. A non-random spin glass with glassy behavior. J. Phys. A: Math. Gen., 27:7646-7668, 1994.

[OLLN02] C. S. O'Hern, S. A. Langer, A. J. Liu, and S. R. Nagel. Random packings of frictionless particles. Physical Review Letters, 88(7), 2002.

[Par14] G. Parisi. Soft modes in jammed hard spheres (I): Mean field theory of the isostatic transition. 2014. arXiv:1403.4413.

[PIM06] A. Percus, G. Istrate, and C. Moore, editors. Computational Complexity and Statistical Physics. Oxford Univ Press, Oxford, 2006.

[Ros58] F. Rosenblatt. The perceptron: A probabilistic model for information storage and organization in the brain. Psychological Review, 65(6):386-408, 1958.

[TS10] S. Torquato and F. H. Stillinger. Jammed hardparticle packings: From kepler to bernal and beyond. Rev. Mod. Phys., 82:2633, 2010.

$\left[\mathrm{TSS}^{+}\right.$05] B. P. Tighe, J. E. S. Socolar, D. G. Schaeffer, W. G. Mitchener, and M. L. Huber. Force distributions in a triangular lattice of rigid bars. Phys. Rev. E, 72:031306, 2005.

[TSVvH10] B. P. Tighe, J. H. Snoeijer, T. J. H. Vlugt, and M. van Hecke. The force network ensemble for granular packings. Soft Matter, 6:2908-2917, 2010.

[vH09] M. van Hecke. Jamming of soft particles: geometry, mechanics, scaling and isostaticity. J. Phys.: Cond. Matt., 22(3):033101, 2009.

[WNW05] M. Wyart, S. R. Nagel, and T. A. Witten. Geometric origin of excess low-frequency vibrational modes in weakly connected amorphous solids. Europhys. Lett., 72(3):486-492, 2005.

[Wya12] M. Wyart. Marginal stability constrains force and pair distributions at random close packing. Phys. Rev. Lett., 109(12):125502, 2012.

[ZJT11] C. E. Zachary, Y. Jiao, and S. Torquato. Hyperuniform long-range correlations are a signature of disordered jammed hard-particle packings. Phys. Rev. Lett., 106:178001, 2011. 